\title{
Predictive value of serological factors, maximal standardized uptake value and ratio of Ki67 in patients diagnosed with non-Hodgkin's lymphoma
}

\author{
JINYUAN LU, YOU WU, BING LI, XIU LUO, WENJUN ZHANG, \\ YU ZENG, JIANFEI FU, AIBIN LIANG and BING XIU \\ Department of Hematology, Shanghai Tongji Hospital, \\ Shanghai Tongji University School of Medicine, Shanghai 200333, P.R. China
}

Received April 2, 2019; Accepted June 22, 2020

DOI: $10.3892 / \mathrm{ol} .2020 .11906$

\begin{abstract}
The present study aimed to determine the prognostic value of serological factors, positron emission tomography/computed tomography maximal standardized uptake value (SUVmax) and the immunohistochemical index ratio of $\mathrm{Ki} 67$ (Ki67\%) for patients diagnosed with non-Hodgkin's lymphoma (NHL). A total of 120 patients with NHL who received regular chemotherapy and underwent serological, radiological and pathological examinations at Shanghai Tongji Hospital between July 2015 and March 2019 were retrospectively analyzed. Spearman's correlation analysis was preformed to describe the associations between different categories of indicators. Kaplan-Meier analysis and log-rank test were used to compare the survival of different subgroups. Receiver operating characteristic curves were generated to assess the predictive value of prominent indicators derived from Cox regression analysis. The results indicated that inflammatory cytokines were strongly associated with tumor burden indicators. The correlation between SUVmax and Ki67\% was significant, and SUVmax of the biopsy site exhibited a stronger association with $\mathrm{Ki67 \%}(\mathrm{P}=0.529, \mathrm{P}<0.001)$ compared with SUVmax of the whole body $(\mathrm{P}=0.395, \mathrm{P}=0.017)$. C-reactive protein (CRP), lactate dehydrogenase (LDH) and interleukin- 6 could differentiate the survival status of patients with NHL, whereas no statistical significance in the estimation of overall survival (OS) was obtained for SUVmax and Ki67\%. SUVmax of the biopsy site had only a limited value in the estimation of progression-free survival (PFS), whereas $\mathrm{LDH}, \beta 2$-microglobulin ( $\beta 2-\mathrm{mg})$ and CRP were independent
\end{abstract}

Correspondence to: Dr Bing Xiu, Department of Hematology, Shanghai Tongji Hospital, Shanghai Tongji University School of Medicine, 389 Xincun Road, Putuo, Shanghai 200333, P.R. China E-mail: xiubing1233@tongji.edu.cn

Key words: non-Hodgkin's lymphoma, inflammatory cytokines, tumor burden indicators, maximal standardized uptake value, ratio of Ki67, prognosis predictors of both OS and PFS with high sensitivity and specificity. Among all indicators, CRP and $\beta 2-\mathrm{mg}$ could predict both survival status and complete remission of patients with NHL, whereas the prognostic value of SUVmax and Ki67\% requires further study and discussion.

\section{Introduction}

Non-Hodgkin's lymphoma (NHL) is a group of malignant lymphohematopoietic tumors mainly occurring in lymphatic organs, including lymph nodes, spleen and thymus. In Asia, NHL frequently displays high grade and aggressive subtypes, which indicates a relatively poor prognosis (1). Currently, histopathology is the most important basis for the diagnosis of NHL $(2,3)$; however, clinicians are usually required to formulate therapeutic strategies before pathological results are available. Therefore, it is necessary to identify patients with poor prognosis and provide them with more comprehensive therapies. Certain blood biochemical indices, including serum inflammatory cytokines, tumor burden indicators, and proportion of absolute lymphocyte to absolute monocyte (4), are of interest to clinicians. However, some clinicians still prefer radiological or pathological examinations since they reflect a more intuitive progress of the disease (5). Whether these non-serological examinations are superior to blood biochemical indices is controversial $(6,7)$. Therefore, it is necessary to perform meticulous comparisons of these indicators.

As the most precise radiographic method to evaluate tumor metabolic activity, ${ }^{18} \mathrm{~F}$-fludeoxyglucoase (FDG) positron emission tomography/computed tomography (PET/CT) is widely applied in tumor staging, grading and therapeutic assessment. The maximal standardized uptake value (SUVmax) is a descriptive parameter to indicate the extent of ${ }^{18} \mathrm{~F}-\mathrm{FDG}$ uptake in normal and tumor tissue, and usually positively correlated with tumor malignancy (8-10). The predictive value of SUVmax in solid tumor has been demonstrated in several studies (11-13), whereas it remains elusive whether SUVmax is instructional in hematologic malignancies. Therefore, further studies are required to determine the role of SUVmax in hematologic malignancies. 
Ki67, also known as antigen Ki67 or MKi67, is associated with cellular proliferation and is only expressed in proliferating cells. The ratio of $\mathrm{Ki} 67(\mathrm{Ki} 67 \%)$ in tumor cells prior to and after chemotherapy is of significance in medication guidance, particularly for the selection of cell-cycle-specific chemotherapeutic drugs (14). At present, no definitive conclusion has been made as to whether $\mathrm{Ki} 67 \%$ has sufficient accuracy in predicting the prognosis of patients with NHL $(15,16)$. Therefore, further studies are required.

The present study provided an elaborate assessment of the relationship among serological factors, SUVmax and Ki67\%, as well as their predictive value regarding the long-term prognosis of patients with NHL.

\section{Materials and methods}

Patients and outcomes. Cases of newly-diagnosed NHL at Shanghai Tongji Hospital (Shanghai, China) between July 2015 and March 2019 were collected and evaluated for age, sex, pathological diagnosis, staging, grading, invasiveness and post-chemotherapy effectiveness. These patients also underwent necessary serological, radiological and pathological examinations. The inclusion criteria were defined as follows: i) The pathological diagnosis was clear and uncontroversial; ii) the patient had received regular chemotherapy and followed up for at least 2 months and iii) at least one radiological or pathological examination was performed. Some patients lack staging or grading information during hospitalization, but with complete serological, radiological and pathological data. For those patients, the data were also considered analyzable. Following removal of information-deficient individuals, 120 patients with valid clinical data were included in the present study. Diagnosis of these patients was performed according to the World Health Organization classification for tumors of hematopoietic and lymphoid tissue (17). The International Prognostic Index (IPI) was calculated before treatment to estimate the prognosis of patients with NHL (18). The patients were staged according to Ann Arbor Staging Classification (19). The patients received regular chemotherapy [cyclophosphamide, doxorubicin, vincristine and prednisone (CHOP); or rituximab-CHOP] and were assessed for effectiveness after four courses of treatment. The criteria of efficacy were defined as follows: i) Complete remission (CR), all clinical and radiological lesions had disappeared, lymph nodes and lumps shrunk to normal size, or the sum of products of greatest diameters (SPD) was reduced by $>75 \%$, tumor markers and biochemical indicators were in the normal range and stable for $\geq 4$ weeks; ii) partial remission (PR), SPD is reduced by $>50 \%$, no new lesion appeared and maintained for $\geq 4$ weeks; iii) stable disease, non-PR and non-progressive disease (PD); and iv) PD, the size of any abnormal lymph node determined prior to treatment increased by $>50 \%$ compared with the previous SPD minimum, or a new lesion appeared during or after treatment. Survival data were also collected in order to determine prognosis-associated factors. Patients were followed up every 3 weeks during chemotherapy and every 3 months after discharge. The last follow-up was in September 2019. Overall survival (OS) time was defined as the interval from trial admission to death of any cause, and progression-free survival (PFS) time was defined as the interval from trial admission to disease progression or death from NHL.

Serological examinations. Serological factors included inflammatory cytokines and tumor burden indicators. The inflammatory cytokines were C-reactive protein (CRP), tumor necrosis factor $\alpha$ (TNF- $\alpha$ ), interleukin-6 (IL-6), interleukin-2 receptor (IL-2R) and interleukin-8 (IL-8), whereas the tumor burden indicators were lactate dehydrogenase $(\mathrm{LDH})$, ferritin and blood $\beta 2$-microglobulin ( $\beta 2-\mathrm{mg})$. TNF- $\alpha$, IL-6, IL-2R and IL- 8 were detected using chemiluminescence with Siemens interleukin test kits (IMMULITE and IMMULITE 1000 TNF- $\alpha /$ IL-6/IL-2R/IL-8; cat. no. LKNF1, LK6P1, LKIP1 and LK8P, respectively.) provided by Siemens AG. Fasting venous blood was obtained from patients on the first day of hospitalization and automatically tested with an Immulete 1000 Analyzer (Siemens AG). $\beta 2-m g$ and ferritin were detected using a radioimmunoassay. CRP was detected using immunoturbidimetry and LDH was measured using a creatine kinase assay. The normal ranges for each indicator were: CRP, $<10 \mathrm{mg} / \mathrm{l}$; TNF- $\alpha,<8.1 \mu \mathrm{g} / \mathrm{l}$; IL-6, <5.9 ng/l; IL-2R, 223-710 U/ml; IL-8, <62 ng/l; LDH, 120-250 U/l; ferritin, 30-400 $\mu \mathrm{g} / \mathrm{l}$ (male), 13-150 $\mu \mathrm{g} / \mathrm{l}$ (female); $\beta 2-\mathrm{mg}$, $0.7-1.8 \mathrm{mg} / \mathrm{l}$.

Radiological examination. All patients signed informed consent forms before PET/CT examination. The patients were required to fast for $\geq 6 \mathrm{~h}$ prior to intravenous infusion of ${ }^{18} \mathrm{~F}$-FDG. Fasting glucose was measured and a dose of ${ }^{18} \mathrm{~F}$-FDG ranging between 4.1 and $10.7 \mathrm{mCi}$ was injected into patients depending on their weight and glucose level. After injection, patients rested for $\geq 40 \mathrm{~min}$ in a comfortable position and PET/CT scanning was performed from the skull to the proximal femur. The images were generated through Siemens Biograph Turepoint 40 PET/CT Imager and reconstructed after attenuation correction. The images were merged through Xeleris software. Subsequently, the reconstructed images were assessed by two experienced nuclear medicine physicians and SUVmax was calculated after the range of interest was drawn. Lesions were diagnosed according to the criteria recommended by the International Group for Imaging Diagnosis of Lymphoma (20). In order to describe the association between SUVmax and Ki67\%, two different types of SUVmax were calculated, including SUVmax of the whole body (SUVmax-overall) and SUVmax of the biopsy site (SUVmax-biopsy). Only incipient PET/CT data were collected, and data after chemotherapy or surgery were automatically excluded.

Pathological examination. Immunohistochemical staining was performed in order to determine Ki67\%. For pretreatment steps, the specimens were fixed with $10 \%$ neutral formaldehyde at room temperature for 24-48 h, embedded in paraffin wax, cut into $3-\mu \mathrm{m}$ thick sections and stained with hematoxylin at room temperature for $5 \mathrm{~min}$ and eosin at room temperature for $1 \mathrm{~min}$. Hematoxylin and eosin were provided by Solarbio Science \& Technology (Beijing) Co., Ltd. For Ki67 immunohistochemical staining, the paraffin sections were stained using Ki67 antibody (cat. no. GT2094; 
1:100; Gene Tech Biotechnology Co., Ltd.) on an automated immunostainer (cat. no. GAS95; Gene Tech Biotechnology Co., Ltd.). The antigen was retrieved by heating with EDTA $\left(\mathrm{pH} \mathrm{9.0)}\right.$ at $100^{\circ} \mathrm{C}$ for $20 \mathrm{~min}$. Blocking was performed using $3 \% \mathrm{H}_{2} \mathrm{O}_{2}$ at $37^{\circ} \mathrm{C}$ for $10 \mathrm{~min}$. The primary antigen was incubated at $37^{\circ} \mathrm{C}$ for $30 \mathrm{~min}$. EnVision reagent (HRP anti-Rabbit/Mouse, cat. no. GK801030, Gene Tech Biotechnology Co., Ltd.) was incubated at $37^{\circ} \mathrm{C}$ for $20 \mathrm{~min}$. The sections were stained by diaminobenzidine (DAB) at room temperature for $8 \mathrm{~min}$, and then stained with hematoxylin at room temperature for $5 \mathrm{~min}$. The nuclei stained with antibody were considered positive, and the number of Ki67-positive tumor cells in 1,000 tumor cells was calculated by 2 experienced pathologists under a light microscope at the 200x magnification. Examples of stained Ki67 sections were shown in Fig. S7. PBS was used as a negative control and Ki67-positive sections were regarded as the positive control.

Statistical analysis. All clinical data were analyzed using SPSS v20.0 software (IBM Corp.). Continuous variables are presented as mean $\pm \mathrm{SD}$, categorical variables are presented as $\mathrm{n}(\%)$. The association between two different indicators was assessed by Spearman's non-parametric correlation analysis. For survival analysis, Kaplan-Meier curves were drawn and the log-rank test was used to compare the survival between groups. $\chi^{2}$ and P-values were calculated to show the extent of significance for survival data. Univariate and multivariate Cox regression analysis was performed to determine prognosis-associated indicators. The regression model was obtained with the method of 'Forward: Wald' (21). Receiver operating characteristic (ROC) curves were depicted to identify the optimal cut-off for indicators, and the area under the curve (AUC) as well as 95\% CI were calculated to determine their predictive value. Youden index (sensitivity + specificity-1) was calculated to find optimal cutoff for those indicators. Mann-Whitney test was used to compare the levels of OS- or PFS-predicting indicators between patients that did or did not achieve CR. $\mathrm{P}<0.05$ was considered to indicate a statistically significant difference.

\section{Results}

Patient characteristics. Among 120 patients aged between 15 and 93 years, 77 (64.2\%) were male and 43 (35.8\%) were female, and their respective average ages were $59 \pm 16$ and $62 \pm 16$ years. The age of all patients, whether grouped by sex or not, was normally distributed. A total of $86(71.7 \%)$ patients were diagnosed with B-cell NHL (B-NHL) and 34 (28.3\%) patients were diagnosed with T-cell NHL (T-NHL). Among them, 104 (86.7\%) were diagnosed with invasive NHL, and $16(13.3 \%)$ were diagnosed with non-invasive NHL. According to Ann Arbor stage ( $n=112)$, most patients were in stage IV (46; 41.1\%), and the numbers of patients in stage I, II and III were $5(4.5 \%), 22(19.6 \%)$ and 39 (34.8\%), respectively. A total of 55 (49.1\%) patients had B symptoms (fever, night sweat or weight loss), whereas the other 57 (50.9\%) patients did not exhibit B symptoms ( $\mathrm{n}=112)$. A total of 44 (41.5\%) patients reached CR after four regular courses of chemotherapy, whereas $62(58.5 \%)$ patients did not
Table I. Characteristics of 120 patients with NHL.

\begin{tabular}{lc}
\hline Characteristic & Value \\
\hline Median age, years (range) & $61(15-93)$ \\
Sex, $\mathrm{n}(\%)$ & \\
Male & $77(64.2)$ \\
Female & $43(35.8)$ \\
Type, $\mathrm{n}(\%)$ & \\
B-NHL & $86(71.7)$ \\
DLBCL & $62(51.7)$ \\
FL & $11(9.2)$ \\
MCL & $6(5.0)$ \\
MALT & $5(4.2)$ \\
Burkitt & $2(1.7)$ \\
T-NHL & $34(28.3)$ \\
AITL & $12(10.0)$ \\
PTCL & $10(8.3)$ \\
NK/T & $4(3.3)$ \\
TLBL & $4(3.3)$ \\
ALCL & $4(3.3)$ \\
Invasiveness, $n(\%)$ & \\
Invasive & $104(86.7)$ \\
Non-invasive & $16(13.3)$ \\
Ann Arbor stage, $\mathrm{n}(\%)^{\mathrm{a}}$ & \\
I & $5(4.5)$ \\
II & $22(19.6)$ \\
III & $39(34.8)$ \\
IV & $46(41.1)$ \\
B symptoms, $\mathrm{n}(\%)^{\mathrm{a}}$ & \\
Yes & $55(49.1)$ \\
No & $57(50.9)$ \\
CR after treatment, $\mathrm{n}(\%)^{\mathrm{b}}$ & \\
Yes & $44(41.5)$ \\
No & $62(58.5)$ \\
\hline
\end{tabular}

${ }^{a} n=112 ;{ }^{b} n=106$. Data are presented as the median (range) or number (percentage). NHL, non-Hodgkin's lymphoma; DLBCL, diffuse large B-cell lymphoma; FL, follicular lymphoma; MCL, mantle cell lymphoma; MALT, mucosa-associated lymphoid tissue lymphoma; AITL, angioimmunoblastic T-cell lymphoma; PTCL, peripheral T-cell lymphoma; NK/T, natural killer/T-cell lymphoma; TLBL, T lymphoblastic lymphoma; ALCL, anaplastic large cell lymphoma; $\mathrm{CR}$, complete remission.

reach CR ( $\mathrm{n}=106)$. The median follow-up time for all patients was 14.5 months (range, 0.6-43.6 months). Details of patient characteristics are provided in Table I.

Correlation analysis. In order to explore the association among different categories of indicators, correlation analysis was performed. This unveiled an association between inflammatory cytokines and tumor burden indicators. LDH was correlated with CRP, IL-6, IL-2R and TNF- $\alpha(\mathrm{P}<0.001)$. $\beta 2-\mathrm{mg}$ and ferritin also exhibited a significant correlation 

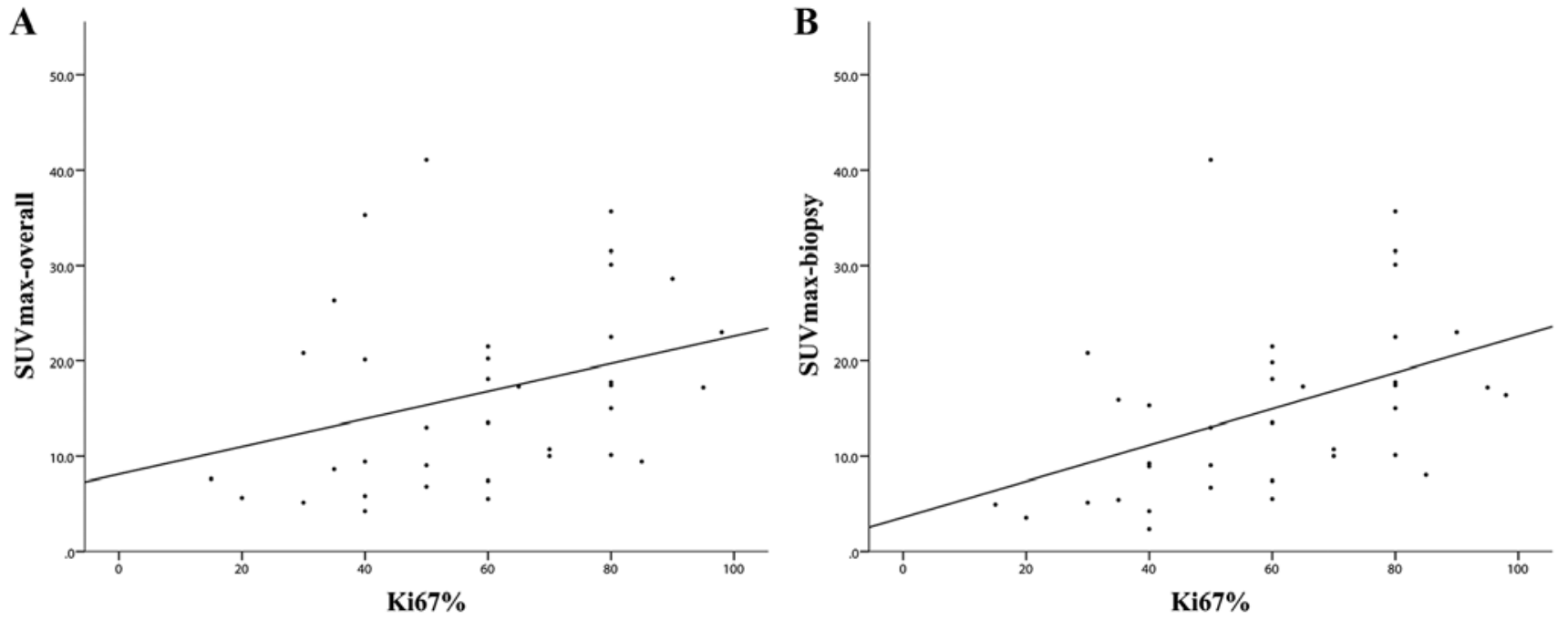

Figure 1. Correlation analysis for SUVmax and Ki67\% in 120 patients with non-Hodgkin's lymphoma. (A) Correlation between SUVmax-overall and Ki67\%; (B) Correlation between SUVmax-biopsy and Ki67\%. Compared with SUVmax-overall, SUVmax-biopsy displayed a better correlation with Ki67\% (P=0.529 for SUVmax-biopsy, compared with P=0.395 for SUVmax-overall). Ki67\%, ratio of Ki67; SUVmax, maximal standardized uptake value.

with these inflammatory cytokines $(\mathrm{P}<0.05)$. However, there was no significant correlation identified between IL-8 and any of the aforementioned tumor burden indicators $(\mathrm{P}>0.05)$. Additionally, LDH, $\beta 2$-mg, IL-6 and IL-2R were associated with age ( $\mathrm{P}<0.05$; data not shown). Furthermore, $\mathrm{LDH}, \beta 2-\mathrm{mg}$, CRP, IL-6, IL-2R and TNF- $\alpha$ exhibited a significant correlation with stage $(\mathrm{P}<0.05$; data not shown). By comparison, there was no significant correlation identified between SUVmax-overall and any of the serological factors $(\mathrm{P}>0.05)$, the same conclusion for SUVmax-biopsy. A noticeable correlation was identified between Ki67\% and ferritin ( $\mathrm{P}=0.235$; $\mathrm{P}=0.020$ ). The correlation between SUVmax and Ki67\% was also significant, and it appeared that SUVmax-biopsy exhibited a stronger association with Ki67\% $(\mathrm{P}=0.529 ; \mathrm{P}<0.001)$ than SUVmax-overall ( $\mathrm{P}=0.395 ; \mathrm{P}=0.017$; Fig. 1; Table II).

Since different subgroups may exhibit different levels of serological, radiological or pathological indexes, a subgroup analysis based on T-/B-NHL or invasive/non-invasive NHL was performed. Compared with when all patients were included, the association between LDH and TNF- $\alpha$ in patients with T-NHL became more significant, but most indicators demonstrated an attenuated correlation (Table SI). For patients with B-NHL, the correlations were similar to the results when all patients were included in the study. Notably, SUXmax-overall was no longer significantly correlated with Ki67\%, whereas the correlation between SUXmax-biopsy and Ki67\% retained its significance (Table SI). When only patients with invasive NHL were included in the analysis, most correlations remained similar (Table SII). Regarding individuals with non-invasive NHL, the sample size was too small for correlation analysis. Therefore, this was not performed.

Survival analysis. To estimate the predictive value of different indicators, patients were automatically stratified into two groups. For serological indicators (CRP, TNF- $\alpha$, IL-6, IL-2R, IL-8, LDH, ferritin and $\beta 2-\mathrm{mg}$ ), the patients were divided into groups according to the normal range of each indicator, with a normal group and an elevated group; For SUVmax and
Ki67\%, patients were divided into groups according to the median value. Based on the Kaplan-Meier survival curves, it became apparent that CRP had the greatest capacity to predict OS of patients with NHL $\left(\chi^{2}=10.124, \mathrm{P}<0.001\right)$. LDH, IL-6 and IL-8 were also prognostic factors $\left(\chi^{2}=9.325, \mathrm{P}=0.002\right.$; $\chi^{2}=4.968, \mathrm{P}=0.026$; and $\chi^{2}=8.507, \mathrm{P}=0.004$, respectively, Fig. 2), whereas $\beta 2-\mathrm{mg}$, ferritin, IL-2R, TNF- $\alpha$, SUVmax and Ki67\% were not statistically significant in OS prediction (data not shown). The subgroup analysis further demonstrated the significance of IL-8 in OS estimation of patients with T-NHL ( $\mathrm{P}=0.038$; Fig. S1). Furthermore, CRP, LDH and IL-6 had the potential to predict OS of patients diagnosed with B-NHL $(\mathrm{P}<0.01$; Fig. S2). For aggressive NHL, CRP, LDH and IL-8 were observed to be significant indicators $(\mathrm{P}<0.05$; Fig. S3), whereas no analysis was performed for non-aggressive NHL due to insufficient sample size.

For PFS, a similar result highlighting the predictive value of CRP $\left(\chi^{2}=11.463 ; \mathrm{P}<0.001\right)$, $\mathrm{LDH}\left(\chi^{2}=7.689 ; \mathrm{P}=0.006\right)$ and IL-6 ( $\left.\chi^{2}=4.366 ; \mathrm{P}=0.037\right)$ was observed (Fig. 3$)$. The subgroup analysis revealed that SUVmax-biopsy was a significant prognostic factor for patients with T-NHL $\left(\chi^{2}=5.266 ; \mathrm{P}=0.022\right.$; Fig. S4). However, none of the other indicators were statistically significant in patients with T-NHL, probably due to limited sample size and heterogeneity of data. By contrast, CRP, LDH and IL-6 had the ability to predict PFS of patients diagnosed with B-NHL, and IL-6 appeared to distinguish PFS of patients with B-NHL more remarkably compared with when all patients were included $\left(\chi^{2}=7.327 ; \mathrm{P}=0.007\right.$; Fig. S5). For aggressive NHL, only CRP was useful for PFS prediction $\left(\chi^{2}=9.632 ; \mathrm{P}=0.002 ; \mathrm{Fig}\right.$. S6), and patients with non-aggressive NHL were automatically excluded due to small sample size.

Univariate and multivariate analysis. Since multiple variables were included in the present study, a univariate analysis followed by a multivariate analysis was performed to determine their predictive value. In Cox regression analysis, multiple variables, including age, sex, aggressiveness, type, stage, B symptoms, IPI score, SUVmax, Ki67\% and all serological factors were set 
Table III. Cox regression analysis for overall survival estimation of 120 patients with non-Hodgkin's lymphoma.

\begin{tabular}{lccccc}
\hline Variables & B & SE & Wald & P-value & RR (95\% CI) \\
\hline LDH & 0.001 & 0.000 & 7.169 & 0.007 & $1.001(1.000-1.002)$ \\
$\beta 2-\mathrm{mg}$ & 0.403 & 0.131 & 9.421 & 0.002 & $1.496(1.157-1.934)$ \\
CRP & 0.024 & 0.010 & 6.358 & 0.012 & $1.024(1.005-1.044)$ \\
IL-8 & 0.037 & 0.011 & 11.025 & 0.001 & $1.037(1.015-1.060)$
\end{tabular}

The regression model contains only variables screened from the univariate analysis. LDH, lactate dehydrogenase; $\beta 2-\mathrm{mg}, \beta 2$-microglobulin; CRP, C-reactive protein; IL-8, interleukin-8; SE, standard error; $\mathrm{RR}$, relative risk.

Table IV. Cox regression analysis for progression-free survival estimation of 120 patients with non-Hodgkin's lymphoma.

\begin{tabular}{lcrrrc}
\hline Variables & B & SE & Wald & P-value & RR (95\% CI) \\
\hline LDH & 0.001 & 0.000 & 11.683 & 0.001 & $1.001(1.000-1.002)$ \\
$\beta 2-\mathrm{mg}$ & 0.211 & 0.070 & 9.110 & 0.003 & $1.235(1.077-1.416)$ \\
CRP & 0.018 & 0.006 & 8.796 & 0.003 & $1.018(1.006-1.030)$ \\
IL-8 & 0.012 & 0.006 & 4.142 & 0.042 & $1.012(1.000-1.023)$
\end{tabular}

The regression model contains only variables screened from the univariate analysis. $\mathrm{LDH}$, lactate dehydrogenase; $\beta 2-\mathrm{mg}, \beta 2$-microglobulin; CRP, C reactive protein; IL-8, interleukin-8; SE, standard error; $\mathrm{RR}$, relative risk.

as independent variables. To perform univariate analysis, each indicator was added to the variable list individually. When entry was set at 0.05 and removal at 0.10 , with the method of 'Forward: Wald', significant indicators, including age $(\mathrm{P}=0.003)$, type $(\mathrm{P}=0.019)$, stage $(\mathrm{P}=0.006)$, B symptoms $(\mathrm{P}=0.004)$, IPI score $(\mathrm{P}<0.001)$, LDH $(\mathrm{P}=0.001), \beta 2-\mathrm{mg}(\mathrm{P}<0.001)$, ferritin $(\mathrm{P}=0.015)$, CRP $(\mathrm{P}<0.001)$ and IL-2R $(\mathrm{P}=0.019)$, were obtained for OS estimation (data not shown). Subsequently, multivariate analysis was performed with the same method and the aforementioned indicators. A regression model containing $\mathrm{LDH}(\mathrm{P}=0.007)$, $\beta 2$-mg $(\mathrm{P}=0.002)$, CRP $(\mathrm{P}=0.012)$ and IL-8 $(\mathrm{P}=0.001)$ was obtained (Table III), indicating that these indicators were independent prognostic factors.

For PFS estimation, univariate analysis revealed age ( $\mathrm{P}=0.011)$, type $(\mathrm{P}=0.004)$, stage $(\mathrm{P}=0.004)$, B symptoms $(\mathrm{P}=0.001)$, IPI score $(\mathrm{P}<0.001), \mathrm{LDH}(\mathrm{P}=0.001), \beta 2-\mathrm{mg}$ $(\mathrm{P}<0.001)$, ferritin $(\mathrm{P}=0.015)$ and $\mathrm{CRP}(\mathrm{P}<0.001)$ as significant indicators (data not shown), and the multivariate analysis demonstrated that LDH $(\mathrm{P}=0.001), \beta 2-\mathrm{mg}(\mathrm{P}=0.003)$, CRP $(\mathrm{P}=0.003)$ and $\mathrm{IL}-8(\mathrm{P}=0.042)$ were significant prognostic indicators (Table IV).

ROC curves. The predictive accuracy of these significant prognostic factors was further evaluated using ROC curves. This revealed that $\beta 2-\mathrm{mg}$ had the highest potential to predict $\mathrm{OS}$ of patients with NHL (AUC, 0.785; 95\% CI, 0.690-0.881; P<0.001; Fig. 4). CRP (AUC, 0.741; 95\% CI, 0.639-0.843; P<0.001) and 

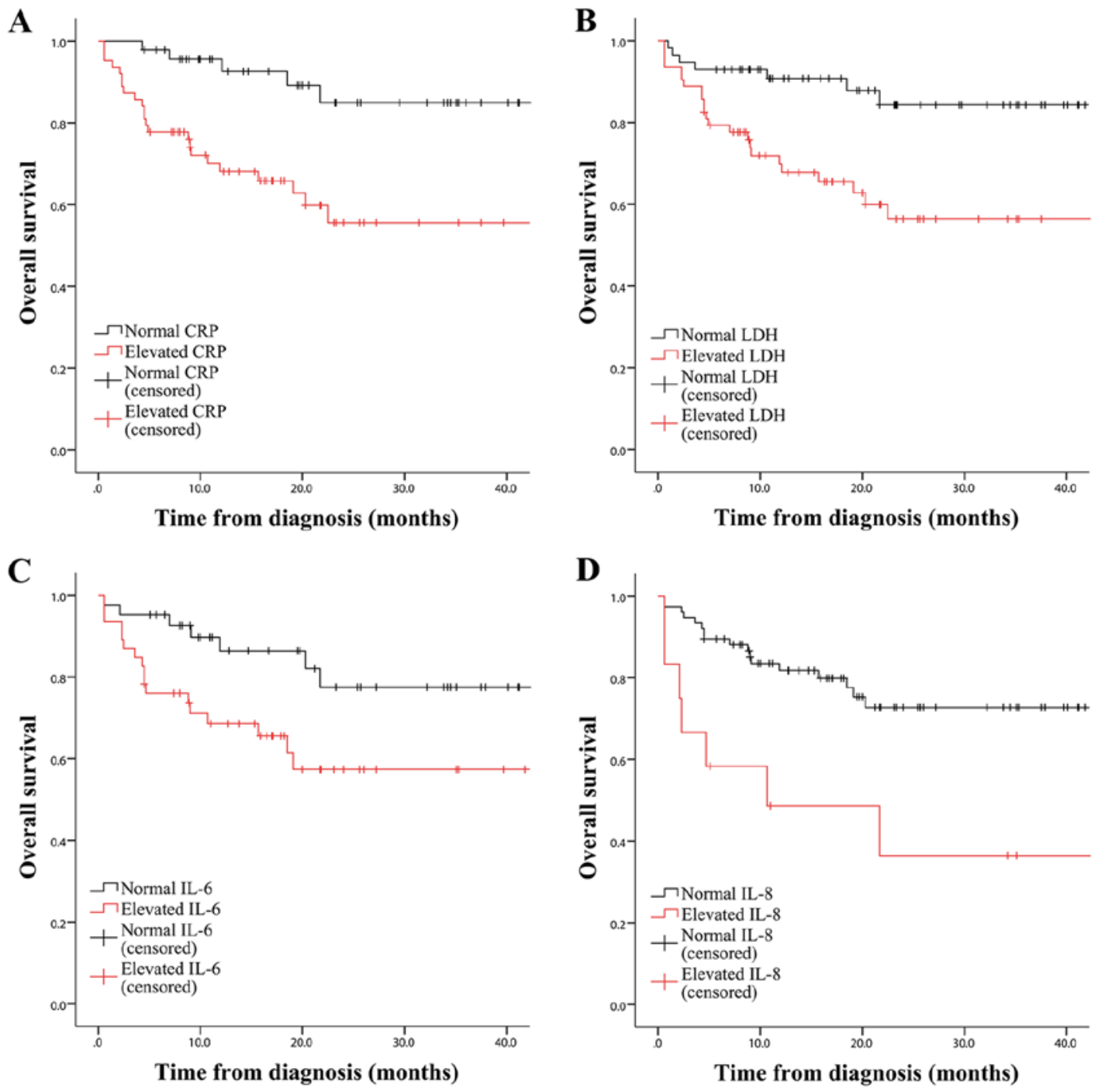

Figure 2. Survival curves of (A) CRP, (B) LDH, (C) IL-6 and (D) IL-8 in overall survival estimation of 120 patients with non-Hodgkin's lymphoma (P<0.001, $\mathrm{P}=0.002, \mathrm{P}=0.026$ and $\mathrm{P}=0.004$, respectively). CRP, C-reactive protein; IL, interleukin; LDH, lactate dehydrogenase.
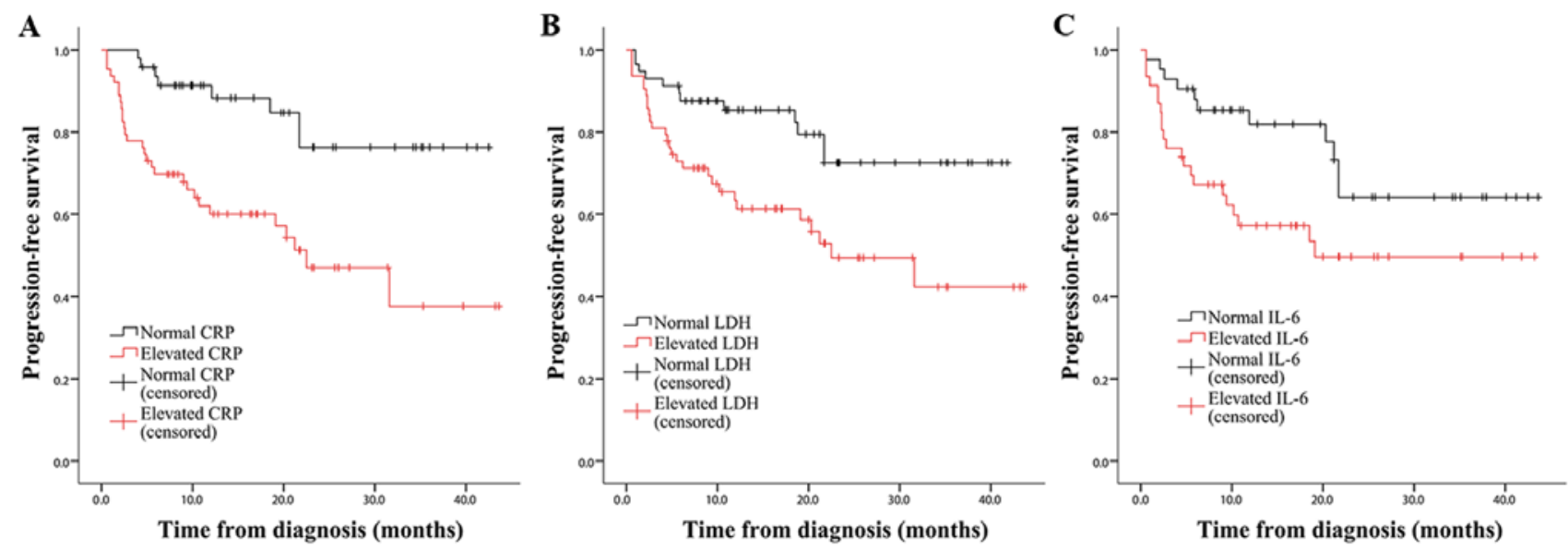

Figure 3. Survival curves of (A) CRP, (B) LDH and (C) IL-6 in progression-free survival estimation of 120 patients with non-Hodgkin's lymphoma (P $<0.001$, $\mathrm{P}=0.006$ and $\mathrm{P}=0.037$, respectively). CRP, C-reactive protein; IL, interleukin; $\mathrm{LDH}$, lactate dehydrogenase.

LDH (AUC, 0.728; 95\% CI, 0.629-0.826; P<0.001) were also indicated to be prognostic factors for OS. As for IL-8, ROC curves did not indicate any significant differences $(\mathrm{P}=0.145$; data not shown), and this was therefore not further discussed. By calculating the Youden Index (sensitivity + specificity-1), the optimal cut-off was determined for these prominent indicators. 

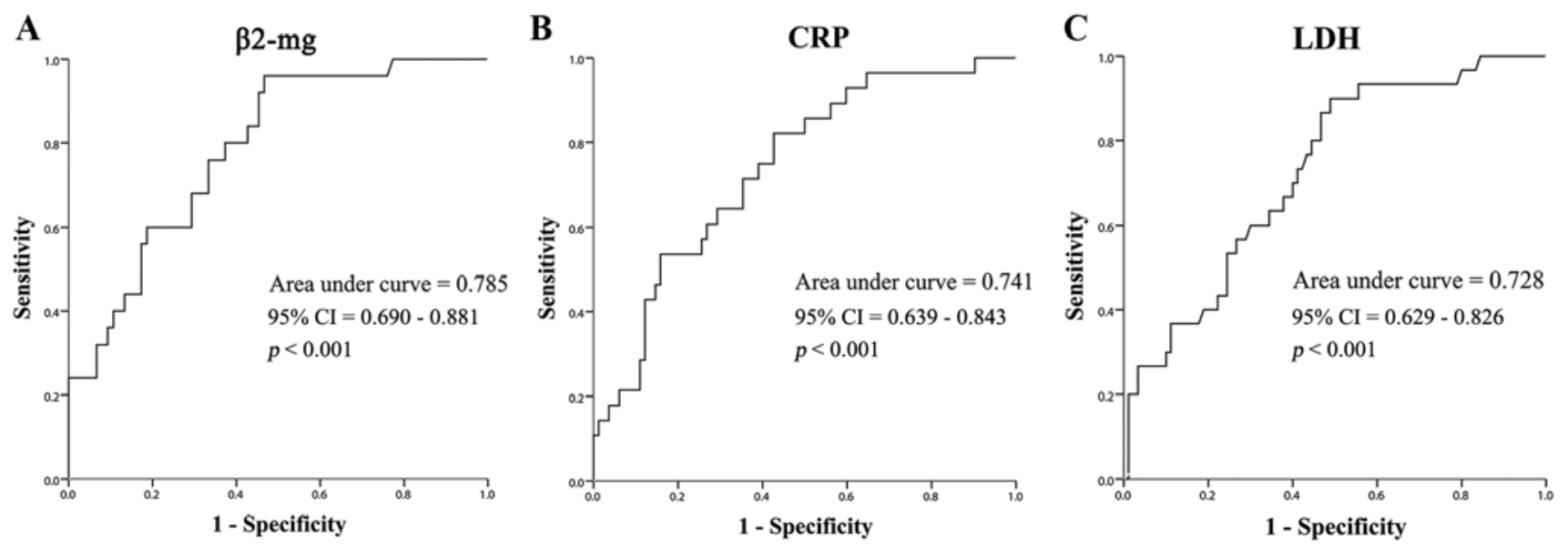

Figure 4. Receiver operating characteristic curves of (A) $\beta 2-\mathrm{mg}$, (B) CRP and (C) LDH in overall survival estimation of 120 patients with non-Hodgkin's lymphoma. $\beta 2$-mg, $\beta 2$-microglobulin; CRP, C-reactive protein; LDH, lactate dehydrogenase.

A

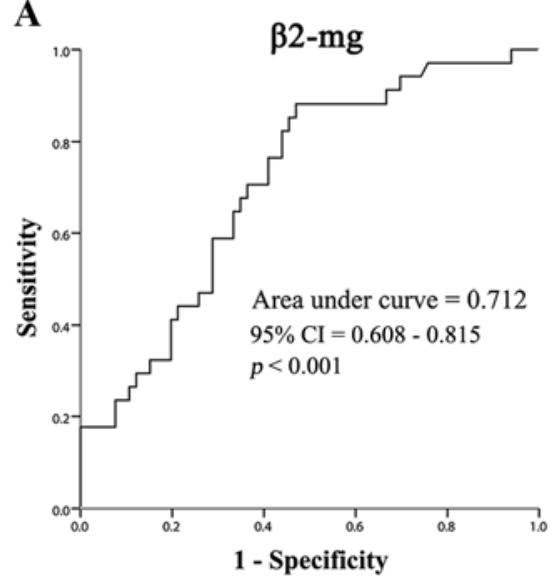

B

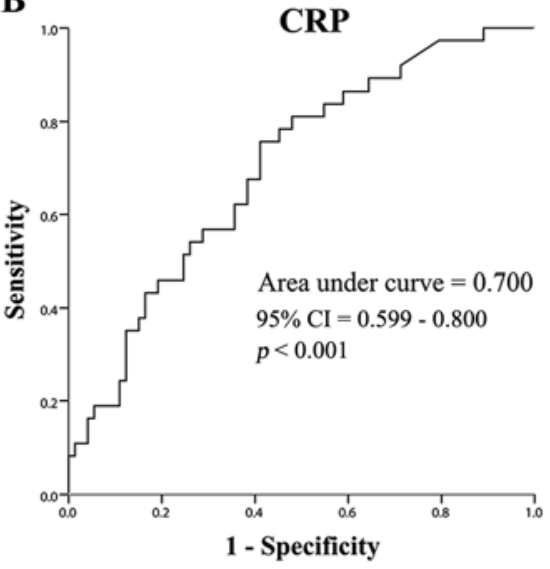

C

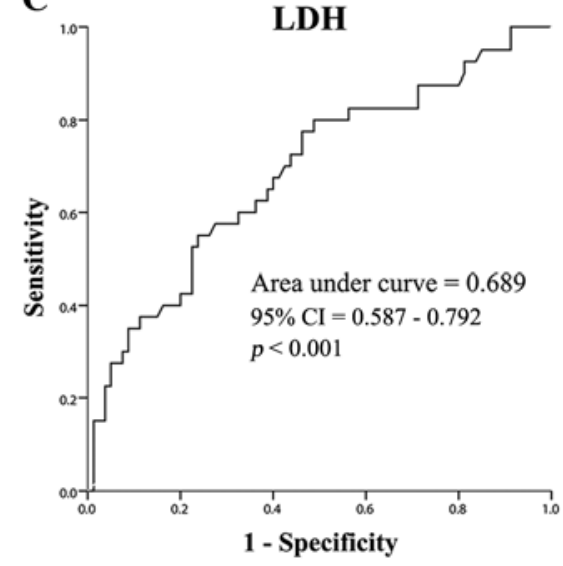

Figure 5. Receiver operating characteristic curves of (A) $\beta 2-\mathrm{mg}$, (B) CRP and (C) LDH in progression-free survival estimation of 120 patients with non-Hodgkin's lymphoma. $\beta 2$-mg, $\beta 2$-microglobulin; CRP, C-reactive protein; LDH, lactate dehydrogenase.

The most suitable cut-off for $\beta 2$-mg with the optimal effectiveness of prediction was $2.89 \mathrm{mg} / \mathrm{l}$ (sensitivity, 0.960; specificity, 0.467), The best cut-off value for LDH to predict OS was $215 \mathrm{U} / 1$ (sensitivity, 0.900; specificity, 0.489). For CRP, the Youden Index reached the maximum when the cut-off was set at $12.01 \mathrm{mg} / 1$ (sensitivity, 0.821; specificity, 0.427) (data not shown).

As for PFS, a similar result demonstrating the predictive potential of $\beta 2-\mathrm{mg}$ (AUC, 0.712; 95\% CI, 0.608-0.815; $\mathrm{P}<0.001$ ), CRP (AUC, 0.700; 95\% CI, 0.599-0.800; $\mathrm{P}<0.001$ ) and LDH (AUC, 0.689; 95\% CI, 0.587-0.792; P<0.001) was observed (Fig. 5), and the optimal cutoff points were set as $2.78 \mathrm{mg} / 1$ (sensitivity, 0.882; specificity, 0.530), $12.01 \mathrm{mg} / 1$ (sensitivity, 0.757; specificity, 0.589) and $224 \mathrm{U} / 1$ (sensitivity, 0.775 ; specificity, 0.538 ), respectively.

CR prediction of $O S$ or PFS indicators. Further investigations were performed to identify whether these serological indicators predicting OS or PFS could predict CR as well. Mann-Whitney U tests demonstrated that patients who reached $\mathrm{CR}$ after four rounds of chemotherapy exhibited significantly lower levels of CRP and $\beta 2-\mathrm{mg}$ at the beginning of treatment compared with those patients who did not achieve $\mathrm{CR}(\mathrm{P}<0.001$; data not shown), indicating that CRP and $\beta 2$-mg were prognostic factors for survival and CR evaluation. Among the other indicators in survival analysis, IL-6 was also a prognostic factor in $\mathrm{CR}$ assessment $(\mathrm{P}<0.001$; data not shown), providing evidence that serological factors have a higher potential to predict CR after four rounds of chemotherapy compared with SUVmax and $\mathrm{Ki} 67 \%$.

\section{Discussion}

With the advantage of intuitively displaying size, location and metabolic activity of malignancies, PET/CT has been widely applied for precise staging and therapeutic evaluation. A higher SUV is indicative of a larger quantity of vigorous and invasive tumor tissue, and a corresponding poor prognosis (8-10). Several studies have demonstrated this relationship in NHL (22,23). In the present study, only a limited significance for SUVmax-biopsy to predict PFS of patients with T-NHL was observed. This may be due to heterogeneity of data, and may not represent the actual situation. A more detailed subgroup analysis based on individual diseases, such as DLBCL, is required for future studies in order to reduce bias. Furthermore, some articles $(24,25)$ have suggested substitution 
of SUVmax with a more responsive indicator, the alteration of SUVmax ( $\triangle$ SUVmax), to evaluate interdependency. However, since repeated PET/CT examination may cause a large financial burden and additional exposure to radiation, not all patients consent to frequent follow-up examinations and clinical data may be difficult to collect. One solution is to calculate SUVmax of the biopsy site (SUVmax-biopsy) instead of the entire body (26). This has been demonstrated in the present study and certain other studies, and a more significant correlation between SUVmax-biopsy and Ki67\% was observed.

Ki67\% is considered to be one of the most useful pathological indicators to reflect tumor proliferation (27). However, in the present study, it was not a significant prognostic factor for NHL. One primary reason is that the definition of Ki67\% is largely subjective and technology-dependent. At times, pathologists tend to overestimate the grade when the percentage of Ki67-positive tumor cells is high. Additionally, the location of the biopsy site affects judgement, and low-quality samples may increase measurement errors and reduce the efficacy of correlation assessment. Previous studies do not provide affirmative answers regarding whether Ki67\% has promising predictive power as opposed to other mainstream predictors $(6,28)$. Perhaps a combination of Ki67\% with other pathological indicators will provide a more suitable option. However, it is certain that the association between SUVmax and Ki67\% is close, which indicates that the metabolic activity of the tumor is associated with its proliferative activity, and this has been demonstrated in multiple studies $(10,29)$.

The present study indicated that traditional serological factors exhibited a higher predictive value than SUVmax and Ki67\%. In the survival analysis, CRP was demonstrated to be the best predictor for both OS and PFS. Additionally, LDH and IL-6 had a marked practicality in the estimation of OS and PFS. By performing Cox regression analysis, it was demonstrated that CRP, LDH and $\beta 2-\mathrm{mg}$ were fully independent predictors of OS and PFS, and their ROC curves indicated high sensitivity and specificity. Furthermore, CRP, $\beta 2$-mg and IL- 6 were significant predictors of $\mathrm{CR}$ after four courses of chemotherapy. This is instructional because clinicians may regard them as key indicators for NHL prognosis and formulate individualized therapies based on their detection level. However, the present study also had some limitations. First, the sample size was relatively small. An increased sample size and extended observation of patient outcome are required in future studies. Second, the number of cases exhibited a great variation among different subgroups. As a result, certain subgroups were too small to be analyzed. Finally, PET data may not be sufficiently adequate to draw firm conclusions. However, our study have demonstrated that serological factors are superior to SUVmax and Ki67\%, which are of clinical importance and have a guidance value.

In conclusion, the present study provided comparisons of serological, radiological and pathological indicators and their ability to predict survival and treatment outcomes for patients with NHL. Overall, it is recommended to use $\beta 2-\mathrm{mg}, \mathrm{CRP}$ and LDH for estimating OS and PFS as the first choice, and taking CRP, $\beta 2-\mathrm{mg}$ and IL-6 into account for CR prediction. CRP and $\beta 2-\mathrm{mg}$ can be used for estimating survival as well as CR. By contrast, SUVmax and Ki67\% did not exhibit sufficient predictive power and should not be recommended. The present study may serve as a reference for clinicians to formulate therapeutic regimens for patients diagnosed with NHL by referring to indicators with high predictive value.

\section{Acknowledgements}

The authors would like to thank Dr Bing Xiu (Department of Hematology, Shanghai Tongji Hospital, Shanghai Tongji University School of Medicine), for her guidance in writing this article, and her support for statistical analysis and polishing the language of the manuscript. The authors would also like to thank Dr Yu Zeng, Dr Bing Li, Miss Yihan Liu and Miss You Wu (Department of Hematology, Shanghai Tongji Hospital, Shanghai Tongji University School of Medicine), who joined in this project and provided reasonable suggestions and necessary support in writing the manuscript.

\section{Funding}

This article was supported by grants from the National Natural Science Foundation (grant nos. 81600156 and 81401882), the clinical research cultivation project of Shanghai Tongji Hospital (ITJ(QN)1907) and the Key Project of Natural Science Foundation of China (grant no. 81830004).

\section{Availability of data and materials}

The datasets generated and/or analyzed during the present study are available from the corresponding author on reasonable request.

\section{Authors' contributions}

JL collected PET/CT data and was a major contributor in writing the manuscript. YZ provided all necessary pathological data including supplementary immunohistochemical photographs. YW was the main collector of serological data and contributed to writing the manuscript. BX revised the manuscript and provided assistance for statistical analysis. BL and XL collected basic information of enrolled patients and collected survival data from patient follow-up. BX, JF, WZ and AL contributed to the conception and design of this study as well as acquisition of funding. All authors have read and approved the final manuscript.

\section{Ethics approval and consent to participate}

All experiments were ethically approved by The Ethics Committee of Shanghai Tongji Hospital (approval no. KYSB-2016-19), and written informed consent was provided by each patient.

\section{Patient consent for publication}

Not applicable.

\section{Competing interests}

The authors declare that they have no competing interests.

\section{References}

1. Müller AM, Ihorst G, Mertelsmann R and Engelhardt $M$ : Epidemiology of non-Hodgkin's lymphoma (NHL): Trends, geographic distribution, and etiology. Ann Hematol 84: 1-12, 2005. 
2. Basharat S, Iqtidar BH, Naeem S, Batool Z, Ali N and Ahmad Z: Clinicopathological and immunohistochemical profile of patients with Non-hodgkin's lymphoma. RMJ 44: 472-476, 2019

3. Iyer VK: Pediatric lymphoma diagnosis: Role of FNAC, biopsy, immunohistochemistry and molecular diagnostics. Indian J Pediatr 80: 756-763, 2013.

4. Binder M, O'Byrne MM, Maurer MJ, Ansell S, Feldman AL Cerhan J, Novak A Porrata LF, Markovic S, Link BK and Witzig TE: Associations between elevated pre-treatment serum cytokines and peripheral blood cellular markers of immunosuppression in patients with lymphoma. Am J Hematol 92: 752-758, 2017.

5. Ansell SM and Armitage J: Non-Hodgkin lymphoma: Diagnosis and treatment. Mayo Clin Proc 80: 1087-1097, 2005.

6. Küçükzeybek BB, Bener S, Çallı AO, Paksoy TD and Payzin B Prognostic significance of Bcl-2 and p53 protein expressions and Ki67 proliferative index in diffuse large B-cell lymphoma. Turk J Haematol 30: 275-282, 2013

7. Fayad L, Keating MJ, Reuben JM, O'Brien S, Lee BN, Lerner S and Kurzrock R: Interleukin-6 and interleukin-10 levels in chronic lymphocytic leukemia: Correlation with phenotypic characteristics and outcome. Blood 97: 256-263, 2001.

8. Cheng G, Servaes S, Alavi A and Zhuang H: FDG PET and $\mathrm{PET} / \mathrm{CT}$ in the management of pediatric lymphoma patients. PET Clin 3: 621-634, 2008

9. Watabe T, Tatsumi M, Watabe H, Isohashi $\mathrm{K}$, Kato $\mathrm{H}$, Yanagawa M, Shimosegawa E and Hatazawa J: Intratumoral heterogeneity of F-18 FDG uptake differentiates between gastrointestinal stromal tumors and abdominal malignant lymphomas on PET/CT. Ann Nucl Med 26: 222-227, 2012.

10. Novelli S, Briones J, Flotats A and Sierra J: PET/CT Assessment of follicular lymphoma and high Grade B cell lymphoma-good correlation with clinical and histological features at diagnosis. Adv Clin Exp Med 24: 325-330, 2015.

11. Berghmans T, Dusart M, Paesmans M, Hossein-Foucher C, Buvat I, Castaigne C, Scherpereel A, Mascaux C, Moreau M, Roelandts M, et al: Primary tumor standardized uptake value (SUVmax) measured on fluorodeoxyglucose positron emission tomography (FDG-PET) is of prognostic value for survival in non-small cell lung cancer (NSCLC): A systematic review and meta-analysis (MA) by the European lung cancer working party for the IASLC lung cancer staging project. J Thorac Oncol 3 : 6-12, 2008.

12. Diao W, Tian F and Jia Z: The prognostic value of SUV (max) measuring on primary lesion and ALN by (18) F-FDG PET or PET/CT in patients with breast cancer. Eur J Radiol 105: 1-7, 2018.

13. Hu SL, Yang ZY, Zhou ZR, Yu XJ, Ping B and Zhang YJ: Role of SUV(max) obtained by 18F-FDG PET/CT in patients with a solitary pancreatic lesion: Predicting malignant potential and proliferation. Nucl Med Commun 34: 533-539, 2013.

14. Tokuda E, Horimoto Y, Arakawa A, Himuro T, Senuma K, Nakai K and Saito M: Differences in Ki67 expressions between pre- and post-neoadjuvant chemotherapy specimens might predict early recurrence of breast cancer. Hum Pathol 63: 40-45, 2017.
15. Gaudio F, Giordano A, Perrone T, Pastore D, Curci P, Delia M, Napoli A, de Risi C, Spina A, Ricco R, et al: High Ki67 index and bulky disease remain significant adverse prognostic factors in patients with diffuse large B cell lymphoma before and after the introduction of rituximab. Acta Haematol 126: 44-51, 2011.

16. Uzurov-Dinić V, Savic A, Lazarević T, Cemerikić-Martinović V, Agić D and Popović S: Prognostic factors in patients with diffuse large B-cell lymphoma. Med Pregl 62: 171-176, 2009.

17. Swerdlow SH: WHOClassification of Tumours of Haematopoietic and Lymphoid, 2008.

18. International Non-Hodgkin's Lymphoma Prognostic Factors Project: A predictive model for aggressive Non-Hodgkin's-Lymphoma. N Engl J Med 329: 987-994, 1993.

19. Carbone PP, Kaplan HS, Musshoff K, Smithers DW and Tubiana M: Report of the Committee on Hodgkin's disease staging classification. Cancer Res 31: 1860-1861, 1971.

20. Juweid ME, Stroobants S, Hoekstra OS, Mottaghy FM, Dietlein M, Guermazi A, Wiseman GA, Kostakoglu L, Scheidhauer K, Buck A, et al: Use of positron emission tomography for response assessment of lymphoma: Consensus of the Imaging Subcommittee of International Harmonization Project in Lymphoma. J Clin Oncol 25: 571-578, 2007.

21. Gigliotti E: Discovering statistics using ${ }_{\text {spss }}$, Second edition. JAN 58: 303, 2007.

22. Lee H, Kim SK, Kim YI, Kim TS, Kang SH, Park WS, Yun $\mathrm{T}$ and Eom HS: Early determination of prognosis by interim 3'-deoxy-3'-18F-fluorothymidine PET in patients with non-Hodgkin lymphoma. J Nucl Med 55: 216-222, 2014

23. Ying Z, Wang X, Song Y, Zheng W, Wang X, Xie Y, Lin N, Tu M, Ping L, Liu W, et al: Prognostic value of interim (18)F-FDG $\mathrm{PET} / \mathrm{CT}$ in diffuse large B-cell lymphoma. Chin J Cancer Res 25: 95-101, 2013.

24. Wen SY, Hua JZ, Li QW, Yan X, Xiang C and Jian-Hua S: Prognostic significance of interim F-18-F-FDG PET/CT SUV reduction associated with Ki67 in patients with diffuse large B-cell lymphoma. Nuclear Sci Techniques 25: 1-7, 2014, 2014.

25. Casasnovas RO, Meignan M, Berriolo-Riedinger A, Bardet S, Julian A, Thieblemont $C$, Vera $P$, Bologna $S$, Brière $J$, Jais JP, et al: SUVmax reduction improves early prognosis value of interim positron emission tomography scans in diffuse large B-cell lymphoma. Blood 118: 37-43, 2011 .

26. Tang B, Malysz J, Douglas-Nikitin V, Zekman R, Wong RH, Jaiyesimi I and Wong CY: Correlating metabolic activity with cellular proliferation in follicular lymphomas. Mol Imaging Biol 11: 296-302, 2009.

27. Scholzen T and Gerdes J: The Ki-67 protein: From the known and the unknown. J Cell Physiol 182: 311-322, 2000.

28. Pătraşcu AM, Rotaru I, Olar I, Pătraşcu Ş Ghiluşi MC, NeamTu SD, Nacea JG and Gluhovschi A: The prognostic role of Bcl-2, Ki67, c-MYC and p53 in diffuse large B-cell lymphoma. Rom J Morphol Embryol 58: 837, 2017.

29. Albano D, Bertoli M, Ferro P, Fallanca F, Gianolli L, Picchio M, Giubbini $\mathrm{R}$ and Bertagna F: 18F-FDG PET/CT in gastric MALT lymphoma: A bicentric experience. Eur J Nucl Med Mol Imaging 44: 589-597, 2017.

This work is licensed under a Creative Commons Attribution-NonCommercial-NoDerivatives 4.0 International (CC BY-NC-ND 4.0) License. 